\section{As relações comunicacionais entre os profissionais de saúde e sua influência na coordenação da atenção}

\section{Communicational relations between healthcare professionals and their influence on coordination of care}

\author{
Las relaciones comunicacionales entre los \\ profesionales de salud y su influencia en \\ la coordinación de la atención
}

\section{Resumo}

O estudo objetiva compreender a influência das relações comunicacionais entre os profissionais da rede de atenção à saúde na coordenação entre níveis assistenciais. Estudo qualitativo com dados de pesquisa multicêntrica internacional Equity-LA II, utilizando o referencial teórico da hermenêutica dialética. Escutaram-se áudios de 15 entrevistas a profissionais ( 7 médicos da atenção primária e 5 da atenção especializada e de 3 apoiadores institucionais da atenção primária) de uma rede municipal no agreste pernambucano, Brasil, em 2016. As categorias mistas foram submetidas à análise de conteúdo. Revelou-se o não reconhecimento da atenção primária como ordenadora do cuidado por quase todos os profissionais e a percepção sobre a coordenação do cuidado desvelou obstáculos relacionados aos desencontros em estabelecer relações dialógicas. O saber sobre o papel do médico da atenção primária é incompleto e sua práxis vista com desconfiança por especialistas, a recíproca não ocorrendo. Percebeu-se uma relação interpessoal não dialógica, pautada na relação assimétrica refletida na postura autoritária do especialista e de inferioridade dos médicos da atenção primária. A base do agir comunicativo refere-se às pretensões de validade e não de poder, que é externo à linguagem, e impossibilita a discussão de razões e argumentos. Evidenciou-se pouca disponibilidade ao diálogo e ao reconhecimento recíproco entre os envolvidos, com interdição da situação comunicativa, em que há simetria de participação. Os resultados revelaram fragilidades comunicacionais, sendo necessárias estratégias que permitam o alcance do entendimento comunicativo entre os profissionais, promovendo o seguimento satisfatório entre níveis assistenciais.
Hylany Bezerra de Almeida 1

Lygia Carmen de Moraes Vanderlei 1

Marina Ferreira de Medeiros Mendes 1,2

Paulo Germano de Frias 1

doi: 10.1590/0102-311X00022020

\author{
Correspondência \\ L. C. M. Vanderlei \\ Instituto de Medicina Integral Prof. Fernando Figueira. \\ Rua dos Coelhos 300, Recife, PE 50070-550, Brasil. \\ lygiacarmen@imip.org.br \\ 1 Instituto de Medicina Integral Prof. Fernando Figueira, Recife, \\ Brasil. \\ 2 Universidade Federal Rural de Pernambuco, Recife, Brasil.
}




\section{Introdução}

A transição demográfica e a sobreposição de perfis epidemiológicos observadas em vários países explicitaram a insuficiência dos sistemas de saúde, incapazes de responder às necessidades assistenciais da população pela fragmentação na organização dos serviços originalmente direcionada às condições agudas 1 . O enfrentamento desse cenário foi previsto na base normativa e legal do Sistema Único de Saúde (SUS), com a constituição de redes de atenção à saúde territorializadas integrando diferentes níveis assistenciais 2. Essas, foram pragmaticamente retomadas em 2010, com a definição de suas diretrizes, fundamentos conceituais e operativos, após o período de priorização da municipalização 2,3.

Por intermédio das redes de atenção são organizadas ações e serviços de variadas densidades tecnológicas para uma assistência contínua e integral 3 , sendo imprescindível para o seu fortalecimento a consolidação da atenção primária à saúde (APS) no atributo de coordenar o cuidado e ordenar o acesso aos outros serviços da rede 4,5. Conexões sólidas entre os serviços da APS e da atenção especializada (AE) possibilitam aos usuários a continuidade de cuidados em tempo oportuno e estabelecimento de saúde adequado às suas necessidades 6,7. A fragilização da coordenação do cuidado ameaça a integralidade da atenção e descaracteriza a APS como "porta de entrada" para a rede de saúde, que passa a ter um significado apenas administrativo 4,8 .

A utilização de várias abordagens metodológicas para a investigação da coordenação entre níveis assistenciais permite esclarecer constructos e conceitos mais abrangentes sobre o fenômeno. A maioria delas adota os desenhos epidemiológicos ou quase experimentais de investigação 9,10,11. Análises sob a perspectiva teórica hermenêutica-dialética não são usuais e podem contribuir para ampliar e aprofundar a compreensão sobre a coordenação. Habermas 12 propôs um novo conceito de racionalidade baseado na comunicação intersubjetiva, o paradigma da comunicação, que diferencia os tipos de ação social em teleológica, estratégica, normativa, dramatúrgica e a ação comunicativa, que ocorre quando se pretende chegar a um acordo em um processo de cooperação entre os atores 13,14.

Uma das ideias centrais da hermenêutica é a de que os indivíduos geralmente entendem-se ou colocam-se em um movimento interior e relacional para o acordo 14 . A dialética tem uma atitude crítica, considerando como base da comunicação relações sociais dinâmicas e antagônicas, existindo dissensos e contradições entre os indivíduos que pertencem a grupos e classes diferentes, que são influenciados por interesses particulares ou coletivos e o momento histórico em que vivem ${ }^{13}$. A hermenêutica e a dialética com suas contribuições e limites podem se complementar na melhora do conhecimento sobre processos sociais complexos como saúde/doença e os fenômenos a eles relacionados 13 .

Segundo Habermas, o entendimento pode ser um mecanismo de coordenação quando os envolvidos na comunicação visam a um acordo em relação às pretensões de validade dos seus atos de fala 12 . Assim, é a racionalidade e os argumentos e não a força ou as influências externas que irão determinar as ações, e em vez de organizações coercitivas haverá as de interação social, comprometidas com a comunicação entre os sujeitos 12,13. Baseando-se na relação entre as pessoas serão construídos os vínculos, que se articulam dentro das instituições, que representam a base para a formação das redes de atenção à saúde 1,15. É a rede de conversação entre os sujeitos, gestores, profissionais e usuários que permite a relação entre a interdependência, a integração e a coordenação 16 .

O objetivo deste estudo é compreender a influência das relações comunicacionais entre os profissionais da rede de atenção à saúde na coordenação entre níveis assistenciais.

\section{Método}

Pesquisa avaliativa de abordagem qualitativa, que buscou compreender aspectos da comunicação entre profissionais e sua relação com a coordenação da atenção entre níveis assistenciais baseando-se no referencial da hermenêutica-dialética 12.

O trabalho utilizou informações da vertente qualitativa da linha de base de pesquisa multicêntrica sobre estratégias para a melhoria da atenção nas redes de saúde da América Latina (Equity-LA II) 17, que analisou os três tipos de coordenação da atenção entre níveis assistenciais 10,18:

(1) Coordenação da informação, em suas duas dimensões: (a) transferência da informação clínica e biopsicossocial entre níveis: existência e disponibilidade de mecanismos adequados que a favoreçam 
e de acesso ágil e oportuno; e (b) utilização das informações sobre os pacientes atendidos em outro nível assistencial para a tomada de decisão dos profissionais envolvidos no cuidado.

(2) Coordenação da gestão clínica, em suas três dimensões: (a) coerência da atenção: existência de objetivos clínicos, exames e tratamentos compartilhados entre os níveis assistenciais, reconciliação de tratamentos, adequação clínica da transferência do paciente e ausência de duplicações; (b) seguimento adequado do paciente: existência do responsável pelo acompanhamento clínico, que é o médico da APS, comunicação com o responsável pelo encaminhamento e garantia de consulta oportuna após a transferência; e (c) acessibilidade entre níveis: assistência continuada, sem interrupções no cuidado.

(3) Coordenação administrativa: circuitos administrativos estabelecidos para a ordenação do acesso e centrais de referências, traduzidos na programação prévia de consultas, exames e outros procedimentos.

A investigação foi realizada em Caruaru, município-polo da Região Agreste e o mais populoso do interior do Estado de Pernambuco, Nordeste brasileiro, distando $138 \mathrm{~km}$ da capital. A rede de serviços de saúde dispõe de 75 centros de APS e 18 da AE, entre centros de saúde, policlínicas, ambulatórios, unidades de pronto atendimento, centro de atenção psicossocial e hospitais. O município foi selecionado por atender os pré-requisitos: (1) dispor de continuidade da assistência entre a APS e AE; (2) prestar serviços a uma população definida; (3) prover serviços em áreas urbanas de baixa renda; e (4) estar disposto a implementar intervenções desenhadas e ter liderança proativa para viabilizar a estratégia.

No presente estudo houve a escuta dos arquivos de áudio das gravações originais dos bancos de dados da pesquisa Equity-LA II, obtidos de entrevistas semiestruturadas cujo roteiro contemplou as categorias da coordenação da atenção. Foram selecionados médicos da APS e da AE que atuavam na atenção clínica aos pacientes e apoiadores institucionais, profissionais que desempenham atividades de planejamento, organização e apoio aos processos de trabalho na rede de saúde. As entrevistas foram aplicadas a 15 profissionais (sete médicos da APS, cinco da AE e três apoiadores institucionais da APS) que atuavam a pelo menos seis meses na rede municipal de saúde, no período de agosto a dezembro de 2016.

As entrevistas foram conduzidas no local de trabalho dos informantes, em ambiente reservado, gravadas em áudio e transcritas na íntegra. Os pesquisadores eram profissionais capacitados, membros da equipe da pesquisa Equity-LA II. Duas das autoras do presente estudo participaram do trabalho de campo como entrevistadora e coordenadora técnica.

O material gerado com base nas entrevistas foi organizado e classificado segundo categoria profissional e nível da atenção (APS e AE), de acordo com as dimensões da coordenação anteriormente descritas. A criação das categorias foi mista, proveniente das dimensões da Equity- LA II e emergentes valendo-se da análise do material coletado, baseada na interpretação das interações que ocorrem por meio do diálogo e que podem resultar na integração entre os indivíduos a depender dos processos linguísticos estabelecidos no sentido de obter entendimento, conforme a Teoria da Ação Comunicativa 12 (Quadro 1).

Realizou-se a análise de conteúdo, desenvolvida em três fases: (1) pré-análise dos áudios dos discursos dos atores; (2) leitura compreensiva das transcrições para apreensão do todo e do seu conteúdo latente, e organização do material com separação de conjuntos dos discursos segundo características dos atores, unidades gramaticais (frases ou parágrafos) e/ou por sequência da narração; e (3) elaboração de categorias empíricas ou unidades de sentido, resultantes da identificação de padrões e dados inter-relacionados a um determinado tema, criadas indutivamente com base nas escutas e nas transcrições originais oriundas das entrevistas e/ou da combinação de ambas 19.

A análise final foi realizada relacionando-se as categorias, dimensões e atributos da coordenação às relações comunicacionais, identificadas nos discursos dos informantes, observando-se minuciosamente o conteúdo das falas, os antagonismos e as contradições que compõem as relações sociais baseadas no referencial teórico da hermenêutica dialética. Foram analisadas as entrevistas necessárias até que os objetivos do trabalho fossem contemplados e os dados obtidos passassem a apresentar redundância ou repetição, não contribuindo mais para o aperfeiçoamento da reflexão.

Os entrevistados foram identificados com códigos para que o anonimato fosse garantido: apoiador institucional (AP); médico (M). O estudo foi aprovado pelo Comitê de Ética em Pesquisa em Seres Humanos do Instituto de Medicina Integral Prof. Fernando Figueira (IMIP), com o parecer no 2.127.664 em 20 de julho de 2017 (CAAE 68376517.5.0000.5201). 


\section{Quadro 1}

Categorias de análise emergentes e do marco teórico da coordenação da atenção entre níveis assistenciais. Caruaru, Pernambuco, Brasil, 2016.

\begin{tabular}{|l|l|}
\hline \multicolumn{2}{|c|}{ CATEGORIAS DE ANÁLISE } \\
\hline Emergentes & Práxis da atenção primária à saúde e da atenção especializada \\
\cline { 2 - 2 } & $\begin{array}{l}\text { Aspectos comunicacionais entre profissionais que facilitam ou dificultam a } \\
\text { coordenação assistencial }\end{array}$ \\
\hline Marco teórico (coordenação da informação) & Transferência de informação clínica e biopsicossocial \\
\cline { 2 - 2 } & Utilização da informação \\
\hline Marco teórico (coordenação da gestão clínica) & Seguimento adequado do paciente \\
\cline { 2 - 2 } & Coerência da atenção \\
\hline Marco teórico (coordenação administrativa) & Circuitos administrativos estabelecidos para a ordenação do acesso \\
\hline
\end{tabular}

\section{Resultados}

As informações analisadas revelaram categorias estabelecidas no marco teórico e as emergidas nas escutas das entrevistas (práxis da APS e AE e falhas na comunicação entre profissionais).

\section{Práxis da APS e AE (Quadro 2)}

Emergiu no discurso de quase todos os entrevistados um conhecimento restrito sobre o papel da APS. A maioria reconheceu sua atuação na prevenção de agravos, identificação/diagnóstico de problemas, acompanhamento mais próximo ao usuário prevenindo complicações, orientando e encaminhando ao médico da $\mathrm{AE}$, quando necessário, à exceção de um médico de cada nível de atenção. Apesar de narrativas sobre a importância da equipe de saúde da família no cuidado, as atividades de promoção à saúde como atribuição da APS foram citadas por apenas um profissional, e o seu papel de coordenadora do cuidado, exclusivamente pelos apoiadores institucionais.

Quase todos os entrevistados conheciam o papel da AE. Os médicos da APS falaram sobre a dificuldade no cumprimento de seu papel de acompanhar os pacientes no território adscrito em decorrência do excesso de famílias cadastradas. Para alguns médicos da AE a APS perdeu um pouco de sua característica, pois não está tão próxima aos pacientes quanto deveria.

\section{Transferência de informação clínica e biopsicossocial (Quadro 3)}

Com relação à instituição e utilização de mecanismos de transferência da informação, dois apoiadores explicaram que foram criados com base na identificação das necessidades pela gestão e apresentados aos profissionais.

Todos os entrevistados identificaram a falta de acesso ágil e oportuno à informação, e a maioria referiu como principais problemas a ausência de mecanismos adequados para este fim e a falta de diálogo entre os profissionais.

\section{Utilização da informação (Quadro 3)}

Para alguns profissionais existem mecanismos para a transferência da informação, mas não são utilizados, são mal preenchidos pelos médicos que os enviam, ou não são consultados pelos que recebem. Os médicos da APS confiam com mais frequência nos especialistas, que os subestimam, porém queixam-se da falta de contrarreferência. 


\section{Quadro 2}

Categorias de análise emergentes da coordenação da atenção entre níveis assistenciais. Caruaru, Pernambuco, Brasil, 2016.

\begin{tabular}{|c|c|}
\hline $\begin{array}{l}\text { CATEGORIAS } \\
\text { DE ANÁLISE }\end{array}$ & ITAÇÕES \\
\hline PS & $\begin{array}{l}\text { "É acompanhar de perto esse paciente, desde a prevenção das doenças, e no caso desses que já têm alguma doença crônica (...). Seja } \\
\text { a função do médico, seja em conjunto à enfermeira, assistentes de saúde, agentes comunitários, pra tá tendo uma visão global desse } \\
\text { paciente, caso haja uma necessidade encaminhar" (M3/AE). } \\
\text { “...Eu não tô a par do funcionamento da atenção básica (...), tô falando é o que acontece comigo aqui. A dificuldade que tem o paciente de } \\
\text { chegar até aqui. De que no meu modo de ver não havia necessidade de tanta burocracia para ele chegar até o oftalmologista" (M5/AE). } \\
\text { “...A atenção básica é a primeira porta de entrada pra enxergar este paciente, (...) ela não só tem esse papel de ficar esperando que o } \\
\text { usuário adoeça e venha procurar o serviço, uma vez que é o local mais importante em se fazer prevenção e promoção à saúde..." } \\
\text { (AP2/APS). } \\
\text { "A atenção básica é a ordenadora do cuidado, (...) deve ser a porta de entrada pros serviços de saúde, principalmente das doenças } \\
\text { crônicas (...). Ela é a ordenadora do serviço, inicia o cuidado, mas nunca deve soltar esse paciente" (AP3/APS). } \\
\text { "A gente tem essa dificuldade de observar pacientes tão de perto, porque são muitos (...) procura manter uma periodicidade, mas nunca é } \\
\text { tão próximo quanto se gostaria" (M7/APS). } \\
\text { “... Não sei se é por causa de mudanças de profissionais, (...) não tenho tido boas respostas em relação aos pacientes. Uma série de } \\
\text { causas, mudança profissional, questão de vagas, de consultas, aí acho que a atenção básica mudou muito sua característica..." (M2/AE). }\end{array}$ \\
\hline Práx & $\begin{array}{l}\text { "Diagnosticar, tratar, encaminhar pra atenção básica, quando a patologia é fácil de tratar, orientando nos cuidados que esse paciente } \\
\text { tem que ter e tratar as exacerbações (...) e oriento sempre o paciente que qualquer intercorrência procure marcar" (M2/AE). } \\
\text { “...Acho que todo mundo exerce direitinho, quando chega lá né? [na AE] (...) o paciente vem, começa a usar a medicação que foi prescrita } \\
\text { lá, as orientações que foram feitas e (...) a grande maioria já vem controladinha, quando volta pra mim..." (M1/APS). }\end{array}$ \\
\hline $\begin{array}{l}\text { Aspectos } \\
\text { comunica- } \\
\text { cionais entre } \\
\text { profissionais } \\
\text { que facilitam a } \\
\text { coordenação } \\
\text { assistencial }\end{array}$ & $\begin{array}{l}\text { "A facilidade dessa coordenação do cuidado se dá pela vontade de cuidar do paciente, pela responsabilização pelo território, pela } \\
\text { conduta" (AP3/APS). } \\
\text { "Acho que o que tem muitas vezes é a boa vontade dos profissionais (...), isso é o facilitador. É o profissional abrir mão da marcação, } \\
\text { pedir que o paciente venha antes, é o coordenador da policlínica que facilita a chegada do paciente, é o gerente que tá lá no nível mais } \\
\text { acima, (...) ter sensibilidade pra abrir algumas exceções..." (M1/AE). } \\
\text { "A regulação facilita, em parte. Porque antigamente o paciente teria que se dirigir à secretaria de saúde pra marcar consulta com o } \\
\text { especialista. Hoje é marcado aqui mesmo no posto de saúde, através de um sistema de computador e de telefone também" (M5/APS). } \\
\text { "O fortalecimento da atenção primária, (...) realmente como ordenadora dos serviços. (...) tem avançado bastante nessa proposta, a } \\
\text { própria implantação do apoio institucional. É você ter a gestão mais próxima das equipes e em relação à interação com a atenção } \\
\text { especializada, estamos trabalhando também de ter o apoio institucional na especializada" (AP2/APS). }\end{array}$ \\
\hline $\begin{array}{l}\text { Aspectos } \\
\text { comunica- } \\
\text { cionais entre } \\
\text { profissionais } \\
\text { que dificultam } \\
\text { a coordenação } \\
\text { assistencial }\end{array}$ & 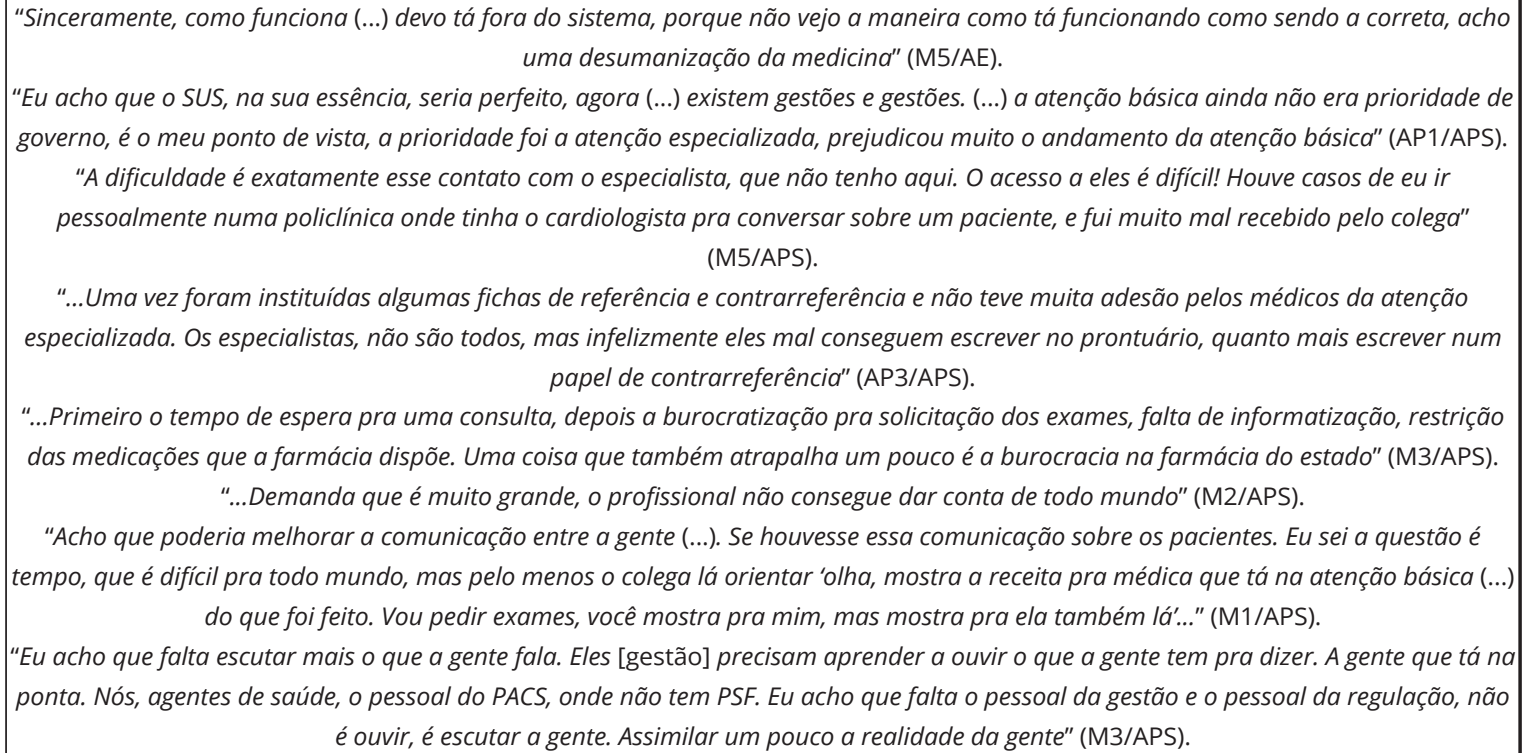 \\
\hline
\end{tabular}

AE: atenção especializada; AP: apoiador institucional; APS: atenção primária em saúde; M: médico; PACS: Programa de Agentes Comunitários de Saúde; PSF: Programa Saúde da Família; SUS: Sistema Único de Saúde. 


\section{Quadro 3}

Categorias de análise do marco teórico da coordenação da informação entre níveis assistenciais. Caruaru, Pernambuco, Brasil, 2016.

\begin{tabular}{|c|c|}
\hline CATEGORIAS DE ANÁLISE & CITAÇÕES \\
\hline Transferência da informação & $\begin{array}{l}\text { "Os mecanismos foram planejados a partir da identificação da gestão, das necessidades, das dificuldades das } \\
\text { unidades. Quem participou foi a gerência de atenção básica no primeiro momento" (AP1/APS). } \\
\text { "A gente teve momentos para ver a apresentação. Ver como funciona. Pra avaliar e dar sugestões, não!" (M7/APS). } \\
\text { "Na verdade, nós mandamos no receituário normal, não tem nada. Nós só botamos ao endocrinologista, ao } \\
\text { pneumologista, e se quiser botamos o motivo, se não, também vai assim. O paciente vai lá e fala" (M6/APS). }\end{array}$ \\
\hline Utilização da informação & $\begin{array}{l}\text { "É um bilhetinho que eu mando, não tem nada de ficha. Eu escrevo por minha conta" (M1/AE). } \\
\text { "A gente não vê exercida a referência e a contrarreferência, (...) a atenção básica meio que fica ilhada, manda o } \\
\text { paciente, ele é atendido, porém não recebe de volta um comunicado do médico, (...) no retorno pra unidade de } \\
\text { saúde é perguntar ao paciente o que foi que o outro médico disse" (AP3/APS). } \\
\text { "Você acha que eu vou pegar meu celular e ficar ligando para discutir sobre paciente? A gente não faz isso na rede } \\
\text { privada! (...) o máximo que vem é uma receita, uma história, um bando de ficha do paciente, (...) às vezes o médico } \\
\text { vê que é muita coisa, papel demais, quer ficar livre para ir embora" (M4/AE). } \\
\text { "...A gente já conhece muitos dos profissionais, tem o celular, liga, tira dúvida. Chefe da pneumologia (...) "olhe } \\
\text { doutora eu tô com uma paciente assim, assim, a senhora atendeu, tô com tal. Não há restrição em esclarecer" } \\
\text { (M3/APS). } \\
\text { "Matriciamento só em saúde mental, (...) mas em todas as outras especialidades não" (M7/APS). } \\
\text { "As unidades de saúde da família reclamam tanto, (...) quanto à necessidade da contrarreferência (...), a gente só } \\
\text { tem instituído em saúde bucal e em atenção ao pré-natal, mas nas outras..." (AP3/APS). } \\
\text { "Não existe muito a contrarreferência. Muito falho (...) os pacientes são encaminhados com toda a referência, eles } \\
\text { não retornam com nada por escrito pra gente. Às vezes ficamos meio perdidos, sem saber o que foi feito lá. Fica } \\
\text { contando só com o esclarecimento do paciente, né?!" (M5/APS). } \\
\text { "Treiná-los a respeito das complicações, da identificação de problemas graves que precisam de um } \\
\text { encaminhamento mais rápido e do tratamento inicial. Dar a eles algumas dicas de como eles poderiam começar a } \\
\text { tratar pra facilitar meu trabalho (...), porque muitas vezes eles não têm noção do que fazer. Os médicos, os clínicos } \\
\text { [médico da APS] que não se reciclam, né?" (M1/AE). }\end{array}$ \\
\hline
\end{tabular}

AE: atenção especializada; AP: apoiador institucional; APS: atenção primária em saúde; M: médico.

Quase todos os entrevistados referiram usar mecanismos informais para transferir informação, como receituários, telefone pessoal ou o paciente como porta voz das suas condutas ao outro nível de atenção, e apenas três afirmaram existir mecanismos formais do matriciamento pontual em saúde mental, atenção à gestante e endocrinologia. Um dos apoiadores explicou que a contrarreferência no município estava instituída apenas para a atenção ao pré-natal e à saúde bucal.

A falha na contrarreferência foi ressaltada por quatro médicos da APS e por três apoiadores como obstáculo ao adequado acompanhamento dos pacientes.

\section{Seguimento adequado do paciente (Quadro 4)}

Um dos aspectos relacionados ao seguimento do paciente é a existência de um responsável clínico, no entanto, apenas dois médicos da APS, um da AE e dois apoiadores identificaram o médico da unidade de saúde da família (USF) como tal, e dois médicos de cada nível não sabiam quem seria o responsável clínico. Para um médico da APS e um apoiador, a responsabilidade é da equipe de saúde da família; para outro médico da APS e dois da AE, é compartilhada entre estes profissionais; e um médico da AE a considera do seu nível de atenção.

Outra perspectiva para o seguimento adequado é a evidência de comunicação oportuna com o responsável pela transição entre níveis e a consulta depois da transferência. Para seis médicos da APS, dois da AE e um apoiador, a comunicação com o médico que transferiu o paciente é falha, os da APS e apoiadores a relacionam ao não envio da contrarreferência, e dois médicos da APS e um da AE 


\section{Quadro 4}

Categorias de análise do marco teórico da coordenação da gestão clínica entre níveis assistenciais. Caruaru, Pernambuco, Brasil, 2016.

\begin{tabular}{|c|c|}
\hline $\begin{array}{l}\text { CATEGORIAS DE } \\
\text { ANÁLISE }\end{array}$ & CITAÇÕES \\
\hline $\begin{array}{l}\text { Seguimento adequado } \\
\text { do paciente }\end{array}$ & 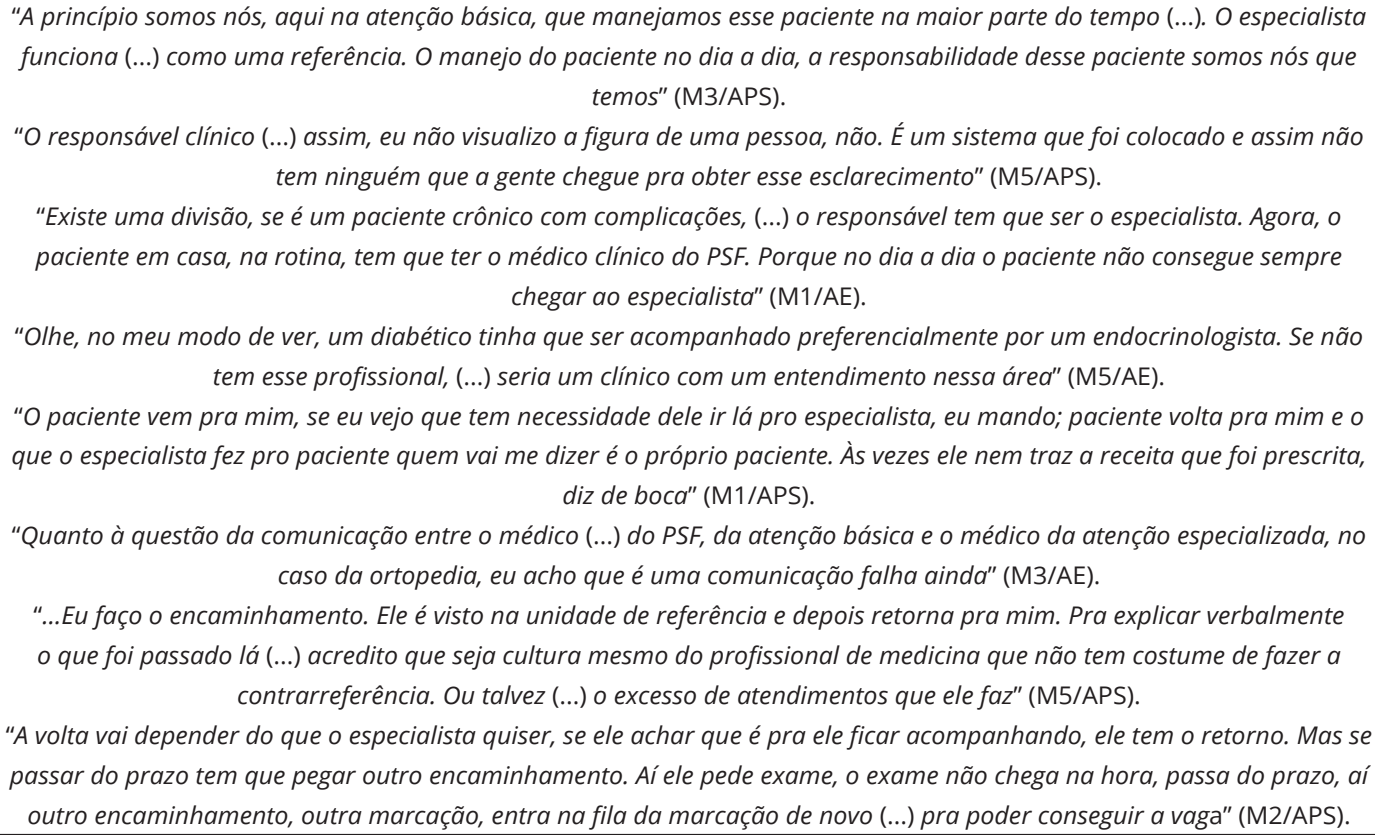 \\
\hline Coerência da atenção & $\begin{array}{l}\text { “Geralmente o médico da atenção básica ele se exime do problema, fica até um pouquinho omisso porque não é } \\
\text { especialista. Pra não se responsabilizar, muitas vezes ele fica mantendo só o que o especialista disse, (...) pra Ihe dar mais } \\
\text { garantia. Então dificilmente tem contradição" (M1/AE). } \\
\text { "Há, há coerência sim. Muitas vezes o que eu faço aqui, o colega lá muda pouca coisa ou então inclui alguma coisa, não há } \\
\text { grande modificação das medicações que são feitas, o tratamento em si que é feito não. Nunca aconteceu não" (M1/APS). } \\
\text { "Algumas vezes têm divergências. E trazem consequências graves. Diagnósticos errados, medicações erradas e isso vai } \\
\text { influenciar muito no paciente" (M2/AE). } \\
\text { “...Há contradição, às vezes ele [o usuário] vai procurar um especialista, que prescreve umas medicações, aí ele não sai } \\
\text { satisfeito, ou não pode comprar as medicações e vai a outro, e já passa outro tipo de medicação” } \\
\text { (M5/APS). } \\
\text { "Não, acontece isso não [duplicação de exames]. Quando eu mando o paciente, eu já mando com os exames, então como } \\
\text { eu já descrevo os exames no encaminhamento, o colega lá já vê, ele vai pedir só algo mais que ele queira” (M1/APS). } \\
\text { "Se ele for atendido, por exemplo, numa emergência, numa UPA, eu não sei por que motivo eles não liberam os exames. O } \\
\text { paciente tem que fazer às vezes novos exames, iguais aos que eles fizeram na unidade” (M5/APS). }\end{array}$ \\
\hline
\end{tabular}

AE: atenção especializada; APS: atenção primária em saúde; M: médico; PSF: Programa Saúde da Família; UPA: unidade de pronto-atendimento.

identificam que o longo tempo de espera para o atendimento dificulta a oportunidade da consulta de seguimento.

\section{Coerência da atenção (Quadro 4)}

Para quatro médicos da APS, três da AE e dois apoiadores, os tratamentos prescritos por médicos dos dois níveis são reconciliados, porém, na visão de dois médicos de cada nível existiam contradições. Foi relatada a inexistência de duplicidades de exames, consultas e medicamentos por dois médicos da AE, um da APS e dois apoiadores, e três médicos da APS e um apoiador perceberam a sua existência. 


\section{Circuitos administrativos estabelecidos para a ordenação do acesso (Quadro 5)}

Quanto aos mecanismos para a coordenação administrativa na rede de saúde, quatro médicos da APS, três da AE e três apoiadores referiram existir uma central de marcação de consultas no município, que para alguns é uma "central de regulação". Um médico da AE relatou usar protocolos de atendimento que não eram do município e um da APS referiu que os fluxos estavam estabelecidos. Dois médicos da APS e um da AE se queixaram da não comunicação com a gestão para a avaliação dos mecanismos.

Outros mecanismos de articulação foram apontados pontualmente pelos médicos e apoiadores, como: prontuário eletrônico, classificação de risco, consultas compartilhadas e matriciamento.

\section{Aspectos comunicacionais entre profissionais que facilitam ou dificultam a coordenação assistencial (Quadro 2)}

O mais frequente aspecto que facilita a coordenação da atenção para dois médicos de cada nível e os três apoiadores é a existência da central de marcação de consultas, além da boa vontade dos profissionais, relatada pontualmente. Apenas os apoiadores citaram o fortalecimento da APS, a execução do seu papel de ordenadora do cuidado e a existência do apoio integrado entre os níveis.

Os aspectos que dificultam a coordenação da atenção para dois apoiadores e um médico da AE foram relacionados ao sistema de saúde; para três médicos da APS e um apoiador, aos profissionais, como a dificuldade de contato com os médicos da AE e de fazerem a contrarreferência, assim como a falta de comunicação da gestão e regulação com os profissionais da assistência. Entretanto, os aspectos dificultadores mais citados (quatro médicos da APS, dois da AE e um apoiador) foram os organizativos, como a "burocracia" para a marcação de consultas, número restrito de vagas para exames, consultas especializadas, medicações e grande demanda de pacientes. A falta de diálogo entre gestão e médicos também foi referida por dois médicos de cada nível de atenção. Apenas para um apoiador havia influências positivas da coordenação na organização da rede e facilidade de acesso.

\section{Quadro 5}

Categorias de análise do marco teórico da coordenação administrativa entre níveis assistenciais. Caruaru, Pernambuco, Brasil, 2016.

\begin{tabular}{|c|c|}
\hline CATEGORIAS DE ANÁLISE & CITAÇÕES \\
\hline $\begin{array}{l}\text { Circuitos administrativos } \\
\text { estabelecidos para a ordenação do } \\
\text { acesso }\end{array}$ & 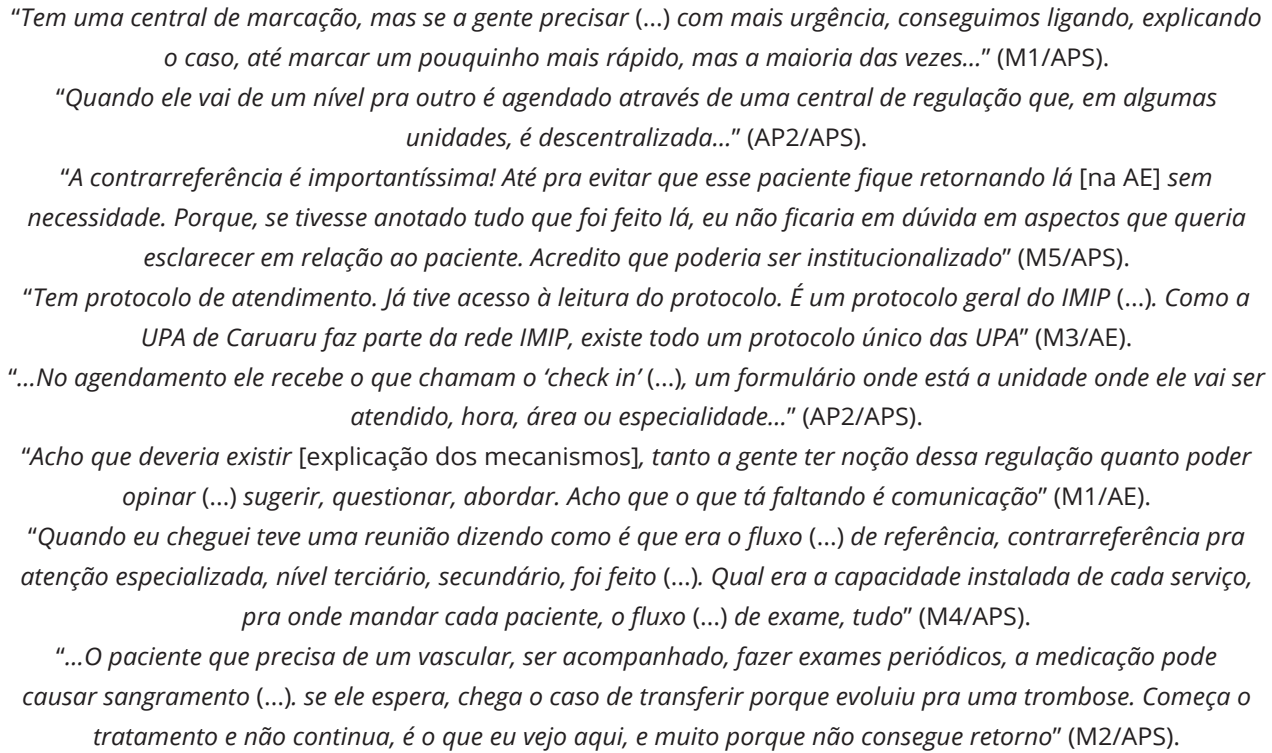 \\
\hline
\end{tabular}

AE: atenção especializada; AP: apoiador institucional; APS: atenção primária em saúde; IMIP: Instituto de Medicina Integral Prof. Fernando Figueira; M: médico; UPA: unidade de pronto-atendimento. 


\section{Discussão}

As noções sobre a coordenação do cuidado na perspectiva dos profissionais de saúde mostraram-se amplas e diversas, assumindo ambivalências e contradições de sentidos. A adoção de um referencial teórico bem estabelecido constituiu o núcleo essencial para explorar a compreensão desses atores, balizada numa perspectiva emergente na tentativa de traduzir as experiências vivenciadas, valores, aspirações, antagonismos e posturas socialmente construídas. Os resultados desvelaram obstáculos impostos à coordenação da atenção e integralidade do cuidado, relacionados aos desencontros no estabelecimento de relações dialógicas entre os profissionais, em que quase todos mostraram um conhecimento restrito sobre o papel da APS. Além dessas barreiras, permaneceram as relativas à organização da rede, como a insuficiência de mecanismos adequados para a articulação e de serviços especializados que garantam o seguimento satisfatório e coerente entre níveis assistenciais.

Apesar do conhecimento de quase todos os entrevistados sobre o papel da APS, independentemente da categoria profissional, este saber é incompleto, não estendido às atividades promocionais de saúde e à sua função primordial de ordenadora do cuidado, percebidas apenas pelos apoiadores institucionais, além de sua práxis na prestação do cuidado, vista com desconfiança por alguns médicos da AE. Por outro lado, a práxis da AE é reconhecida por todos os profissionais, que contam com a confiança dos colegas da APS, apesar destes compreenderem a não reciprocidade. Percebe-se que a relação interpessoal não foi dialógica e sim pautada em um saber superior (AE) em contraposição ao reconhecimento do outro (APS), refletindo nos profissionais da AE uma postura autoritária, não reflexiva e sem a autocrítica ou liberdade crítica genuína, com importantes repercussões na atenção ao usuário $14,20,21$.

Os relatos revelaram semelhança com entrevistas a usuários da rede de dois municípios paulistas 22, que apontaram a impotência das equipes da APS na coordenação do cuidado, seja pela insuficiência de condições materiais, tecnológicas, operacionais e organizacionais, ou as relacionadas aos valores, significados e representações no cumprimento do seu papel, situações compartilhadas no dia a dia dos médicos da APS entrevistados 23. Essa impotência da APS na coordenação do cuidado pode estar relacionada ao insuficiente conhecimento de profissionais da AE sobre o trabalho da APS e o desvalor destes, interferindo na colaboração entre os níveis, o que também foi observado na Espanha 24.

Para a primeira dimensão do núcleo essencial do referencial teórico 18, a coordenação da informação, observou-se falha de articulação entre a APS e AE vivenciada pelo distanciamento entre níveis assistenciais nos quais os profissionais não têm conhecimento sobre quem encaminhou os pacientes, nem acesso às informações da assistência prestada anteriormente. Essa percepção de distanciamento e a necessidade de aproximação emergiu com maior intensidade no relato dos médicos da APS em estudo prévio 6 .

A integração entre a APS e AE pode ser favorecida pela utilização dos mecanismos de articulação entre níveis, como o formulário de referência e contrarreferência. Tais mecanismos existem em um estudo com 16 países da América Latina 25, sendo, entretanto, mais eficientes na referência de pacientes do primeiro ao segundo níveis de atenção, similar ao relato dos entrevistados deste trabalho. Em grandes centros brasileiros, verificou-se que a contrarreferência não era uma prática comum 26, variando de 2,8\% em Belo Horizonte (Minas Gerais) a 11,5\% em Florianópolis (Santa Catarina), semelhante aos resultados revelados por nossos entrevistados e nos cinco outros países pesquisados na Equity-LA II 27, mostrando a necessidade de maior integração entre APS e AE.

Os profissionais afirmaram que a não utilização do mecanismo de referência e contrarreferência é decorrente da falta de tempo e/ou interesse dos especialistas. Dentre os principais motivos para a não utilização da contrarreferência, um estudo desenvolvido no Nordeste brasileiro mostrou a frágil articulação da rede, a deficiência de tecnologias para comunicação, insuficiente conscientização, hábito e empenho dos profissionais 28 .

O desconhecimento dos profissionais sobre o seu papel e a desconfiança dos médicos da AE em relação aos da APS ficou evidente nos discursos de número expressivo dos informantes, indo de encontro do modelo de atenção em redes vigente no país, que preconiza a articulação entre níveis assistenciais para dar respostas às necessidades dos usuários 1,3. Isso induziu a permanência desnecessária dos pacientes na $\mathrm{AE}$, gerando filas de espera e dificultando o acesso a este nível, também referido em estudo anterior 6 . 
Em alguns discursos ficaram evidentes a falta de respeito e de colaboração mútua entre médicos nos dois níveis, com reflexos sobre a comunicação, que reconhece a importância de práticas dialógicas para o estabelecimento de relações mais equitativas e de confiança 21,29.

Percebeu-se a pouca disponibilidade ao diálogo e à intenção de que esta prática seja estabelecida ou de que seja necessária, principalmente na visão dos especialistas, e prevaleceu a sensação de inferioridade em profissionais da APS, refletida no compartilhamento de pacientes. A base para o agir comunicativo refere-se às pretensões de validade e não de poder, que é externo à linguagem, impossibilitando a discussão de razões e argumentos 12,13,14. As condições de diálogo sucedem dos papéis atribuídos aos participantes decorrentes da interseção entre poder e linguagem, existindo diálogo quando há uma troca mútua de perguntas e respostas. Para a existência de uma situação comunicativa é necessária a simetria de participação, com direitos iguais e ausência de coação, o que não é possível sem o reconhecimento recíproco entre os participantes $12,13,14$.

A insuficiente comunicação foi percebida pelos profissionais da gestão e médicos dos dois níveis, apesar de um dos aspectos primordiais para o exercício profissional adequado entre níveis assistenciais ser o estado de espírito cooperativo e de confiança entre os envolvidos 14,29.

A falta da recomendação dos especialistas aos pacientes para informar à atenção básica sobre o seu estado de saúde e condutas, garantindo a continuidade do cuidado sugerida pelo estudo, é outra evidência que influencia na qualidade da atenção. O cuidar da saúde requer encontros entre subjetividades, entre o cuidador e o ser cuidado, desvendando necessidades de saúde como também do que se considera a "boa vida" e como buscá-la 15,21. O caráter técnico-científico do saber por vezes exclui qualquer outro, tornando o encontro assistencial tecnicista e dominador, evidenciando uma assimetria de poder entre o profissional e o paciente, que assume uma condição de objeto a ser transformado pelo que presta o cuidado; esta forma de encontro foi percebida pelos médicos da APS como sendo comum entre especialistas e pacientes $20,21,29$. O estabelecimento de vínculos, essenciais para a qualidade das relações que envolvem o cuidar, depende da construção de oportunidades de encontros que favoreçam intersubjetividades e promovam compartilhamentos 20,21,29.

Os entrevistados perceberam a necessidade de mais encontros e de estímulo à comunicação entre eles para a melhoria da coordenação, tais encontros, se estabelecidos entre os profissionais de diferentes níveis de atenção, possibilitam construir maneiras de lidar com saberes e realizar a tarefa do atendimento integral em saúde que envolve a interação constante de trabalhadores para a efetiva assistência 29. Estudos reconhecem que a comunicação, o conhecimento e a relação entre os profissionais são determinantes centrais da coordenação, influenciando na continuidade da atenção e qualidade do cuidado 30,31,32.

Os profissionais referiram apenas a existência de uma central de marcação de consultas, a utilização de protocolos que não eram do município e, pontualmente, outros mecanismos para a coordenação administrava. Em seus discursos perpassou a compreensão de que os fluxos não estão bem estabelecidos. Essas características geram dificuldades de acesso a consultas especializadas e exames, como apontado em outros estudos 26,32,33, revelando a fragmentação da rede de saúde com barreiras assistenciais ao percurso do usuário, ou ainda, a insuficiência de serviços especializados para a demanda crescente 27,34,35. Por outro lado, estudos com gestores identificaram que a competência técnica e o perfil de profissionais da APS “induzem” encaminhamentos, dificultando a otimização da distribuição de consultas e exames, agregando obstáculos de acesso aos especialistas 28 .

Estudos indicam que o uso de metodologia participativa é especialmente relevante por possibilitar oportunidades de diálogo e colaboração mútua entre profissionais da APS e da AE 9,32,36. Mecanismos como sessões clínicas compartilhadas para análise de casos concretos, estruturas matriciais com proposta de intervenção pedagógico-terapêutica, criação de grupos de trabalho entre APS, AE e representantes da gestão são exemplos de experiências que contribuem para melhorar a comunicação e a qualidade da atenção $36,37,38$.

Entre as limitações do estudo, a escuta de áudios originados de entrevistas com roteiro não elaborado com base nos pressupostos da hermenêutica crítica implicou o esforço interpretativo adicional para minimizar esta lacuna. A busca de um sentido atribuído aos aspectos comunicacionais da coordenação entre níveis vislumbrando os antagonismos e as relações de poder subjacentes foi um imperativo nos constructos de terceira ordem. Procedeu-se a um diálogo constantemente reinterpretado com base no que foi emergindo à medida em que se aprofundava no objeto do estudo, na tentativa de 
fugir do relativismo e garantir a aproximação com a verdade. Por outro lado, o limite imposto a este trabalho foi minimizado ao utilizarmos a mediação conceitual do referencial teórico da coordenação da atenção e da triangulação dos pesquisadores para garantir a validade interna.

Evidenciou-se pouca disponibilidade ao diálogo e ao reconhecimento recíproco entre os profissionais envolvidos, com interdição da situação comunicativa, em que há simetria de participação. Os resultados revelaram fragilidades comunicacionais, sendo necessárias estratégias que permitam o alcance do entendimento comunicativo entre os profissionais, promovendo o seguimento satisfatório entre níveis assistenciais.

\section{Colaboradores}

H. B. Almeida, L. C. M. Vanderlei e M. F. M. Mendes participaram de todas as etapas da autoria do manuscrito nos seguintes termos: concepção, planejamento, análise e interpretação dos dados, redação, revisão crítica do conteúdo e aprovação da versão final. P. G. Frias participou das seguintes etapas da autoria do manuscrito: concepção, interpretação dos dados, redação, revisão crítica do conteúdo e aprovação da versão final.

\section{Informações adicionais}

ORCID: Hylany Bezerra de Almeida (0000-00015596-4497); Lygia Carmen de Moraes Vanderlei (0000-0002-3610-3699); Marina Ferreira de Medeiros Mendes (0000-0002-5752-5217); Paulo Germano de Frias (0000-0003-4497-8898).

\section{Referências}

1. Mendes EV. As redes de atenção à saúde. Brasília: Organização Pan-Americana da Saúde; 2011.

2. Albuquerque AC, Cesse EAP, Felisberto E, Samico IC, Frias PG. Avaliação de desempenho da regionalização da vigilância em saúde em seis Regiões de Saúde brasileiras. Cad Saúde Pública 2019; 35 Suppl 2:e00065218.

3. Ministério da Saúde. Portaria no 4.279, de 30 de dezembro de 2010. Estabelece diretrizes para a organização da Rede de Atenção à Saúde no âmbito do Sistema Único de Saúde (SUS). Diário Oficial da União 2010; $31 \mathrm{dez.}$

4. Bousquat A, Giovanella L, Fausto MCR, Medina MG, Martins CL, Almeida PF, et al. A atenção primária em regiões de saúde: política, estrutura e organização. Cad Saúde Pública 2019; 35 Suppl 2:e00099118.

5. Haggerty J, Reid R, Freeman GK, Starfield $\mathrm{BH}$, Adair CE, McKendry R. Continuity of care: a multidisciplinary review. BMJ 2003; 327:1219-21.

6. Tesser CD, Poli Neto P. Atenção especializada ambulatorial no Sistema Único de Saúde: para superar um vazio. Ciênc Saúde Colet 2017; 22:941-51.

7. Terraza-Núñez R, Vargas-Lorenzo I, VázquezNavarrete ML. La coordinación entre niveles asistenciales: una sistematización de sus instrumentos y medidas. Gac Sanit 2006; 20:48595.

8. Carneiro MSM, Melo DMS, Gomes JM, Pinto FJM, Silva MGC. Avaliação do atributo coordenação da atenção primária à saúde: aplicação do PCATool a profissionais e usuários. Saúde Debate 2014; 38 (n.esp.):279-95.

9. Vargas I, Vázquez ML, Henao D, de Campos C. Implantación de mecanismos de coordinación asistencial en Redes Integradas de Servicios de Salud. Barcelona: Consorci de Salut i Social de Catalunya/Organización Panamericana de la Salud; 2011.

10. Aller MB, Vargas I, Coderch J, Calero S, Cots F, Abizanda M, et al. Development and testing of indicators to measure coordination of clinical information and management across levels of care. BMC Health Serv Res 2015; 15:323. 
11. Breimaier HE, Halfens RJ, Lohrmann C. Effectiveness of multifaceted and tailored strategies to implement a fall-prevention guideline into acute care nursing practice: a before-and-after, mixed-method study using a participatory action research approach. BMC Nurs 2015; $14: 18$.

12. Habermas J. Teoria de la acción comunicativa. Tomo I. 2a Ed. Madrid: Taurus; 1988.

13. Habermas J. Consciência moral e agir comunicativo. Rio de Janeiro: Tempo Brasileiro; 2003.

14. Gadamer H. Verdade e método. Petrópolis: Editora Vozes; 1999.

15. Ayres JRCM. O cuidado, os modos de ser (do) humano e as práticas de saúde. Saúde Soc 2004; 13:16-29.

16. Lima JC, Rivera FJU. Agir comunicativo, redes de conversação e coordenação em serviços de saúde: uma perspectiva teórico-metodológica. Interface (Botucatu) 2009; 31:329-42.

17. Vázquez ML, Vargas I, Unger JP, Paepe P, Mogollón-Pérez AS, Samico I, et al. Evaluating the effectiveness of care integration strategies in different healthcare systems in Latin America: the EQUITY-LA II quasi-experimental study protocol. BMJ Open 2015; 5:e007037.

18. Reid R, Haggerty J, McKendry R. Defusing the confusion: concepts and measures of continuity of healthcare. Ottawa: Canadian Health Services Research Foundation; 2002.

19. Gomes R. Análise e interpretação de dados de pesquisa qualitativa. In: Minayo MCS, organizadora. Pesquisa social: teoria, método e criatividade. Petrópolis: Editora Vozes; 2016. p. 72-95.

20. Ayres JRCM. Para comprender el sentido práctico de las acciones de salud: contribuciones de la Hermenéutica Filosófica. Salud Colect 2008; 4:159-72.

21. Schraiber LB. Quando o 'êxito técnico' se recobre de 'sucesso prático': o sujeito e os valores no agir profissional em saúde. Ciênc Saúde Colet 2011; 16:3041-2.

22. Cecílio LCO, Andreazza R, Carapinheiro R, Araújo EC, Oliveira LA, Andrade MGG, et al. A atenção básica à saúde e a construção das redes temáticas de saúde: qual pode ser o seu papel? Ciênc Saúde Colet 2012; 17:2893-902.

23. Reis DO, Cecílio LCO, Andreazza RAEC, Correia T. Nem herói, nem vilão: elementos da prática médica na atenção básica em saúde. Ciênc Saúde Colet 2018; 23:2651-60.

24. Giovanella L, Escorel S, Lobato LVC, Noronha JC, Carvalho AI, organizadores. Políticas e sistema de saúde no Brasil. 2a Ed. Rio de Janeiro: Editora Fiocruz; 2013.

25. Fernández MA, Roo JP, Irigogen AC, Blanco SL, Edward AB, Juárez VT. Los sistemas de referencia y contrarreferencia de pacientes em América Latina: mecanismos de coordinación asistencial y el rol de la medicina familiar e comunitaria. Rev Bras Med Fam Comunidade 2016; 11 Suppl 2:37-45.

26. Almeida PF, Marin J, Casotti E. Estratégias para consolidação da coordenação do cuidado pela atenção básica. Trab Educ Saúde 2017; 15:373-98.
27. Vargas I, Garcia-Subirats I, Mogollón-Pérez AS, Mendes MFM, Eguiguren P, Cisneros AI, et al. Understanding communication breakdown in the outpatient referral process in Latin America: a cross-sectional study on the use of clinical correspondence in public healthcare networks of six countries. Health Policy Plan 2018; 33:494-504.

28. Aleluia IRS, Medina MG, Almeida PF, Villasboas ALQ. Coordenação do cuidado na atenção primária à saúde: estudo avaliativo em município sede de macrorregião do nordeste brasileiro. Ciênc Saúde Colet 2017; 22:1845-56.

29. Ayres JRCM. Organização das ações de atenção à saúde: modelos e práticas. Saúde Soc 2009; 18 Suppl 2:11-23.

30. Souza CM. Coordenação, uniformidade e autonomia na formulação de políticas públicas: experiências federativas no cenário internacional e nacional. Cad Saúde Pública 2019; 35 Suppl 2:e00046818.

31. Jesus RPFS, Espírito Santo ACG, Mendes MFM, Samico IC. Health care professionals perceptions of coordination between levels of care in two large municipalities in the State of Pernambuco, Brazil. Interface (Botucatu) 2018; 22:423-34

32. Vargas I, Mogollón-Pérez AS, Paepe P, Silva MRF, Unger JP, Vázquez ML. Barriers to healthcare coordination in market-based and decentralized public health systems: a qualitative study in healthcare networks of Colombia and Brazil. Health Policy Plan 2016; 31:73648.

33. Oliveira CRF, Samico IC, Mendes MFM, Vargas I, Vázquez ML. Conhecimento e uso de mecanismos para articulação clínica entre níveis em duas redes de atenção à saúde de Pernambuco, Brasil. Cad Saúde Pública 2019; 35:e00119318.

34. Santos CM, Barbieri AR, Gonçalves CCM, Tsuha DH. Avaliação da rede de atenção ao portador de hipertensão arterial: estudo de uma região de saúde. Cad Saúde Pública 2017; 33:e0005281.

35. Miranda-Mendizábal A, Vargas I, MogollónPérez AS, Eguiguren P, Mendes FMM, López $\mathrm{J}$, et al. Conocimiento y uso de mecanismos de coordinación clínica de servicios de salud de Latinoamérica. Gac Sanit 2020; 34:340-9.

36. Blevins D, Farmer MS, Edlund C, Sullivan G, Kirchner JE. Collaborative research between clinicians and researchers: a multiple case study of implementation. Implement Sci 2010; 5:76.

37. O'Malley AS, Reschovsky JD. Referral and consultation communication between primary care and specialist physicians finding common ground. Arch Intern Med 2011; 171:56-65.

38. Vermeir P, Vandijck D, Degroote S, Peleman S, Verhaeghe R, Mortier E, et al. Communication in healthcare: a narrative review of the literature and practical recommendations. Int J Clin Pract 2015; 69:1257-67. 


\section{Abstract}

The study aims to understand the influence of communicational relations among healthcare professionals in the coordination of care between levels. This is a qualitative study with data from the international multicenter study Equity-LA II, with dialectic hermeneutics as the theoretical reference. The authors listened to the audios from 15 interviews with professionals ( 7 physicians from primary care and 5 from specialized care, and 3 institutional supporters from primary care) in a municipal network in the Agreste region of the state of Pernambuco, Brazil, in 2016. The mixed categories were submitted to content analysis. The analysis revealed a lack of recognition, by nearly all of the professionals, of primary care as the organizational backbone for care, and the perception of coordination revealed obstacles related to disconnects in establishing dialogical relations. Knowledge of the physician's role in primary care is incomplete, and its praxis is viewed with distrust by specialists, while the reciprocal is not true. There was a visibly non-dialogical interpersonal relationship, based on asymmetric relations reflected in the specialist's authoritarian stance and that of inferiority of primary care physicians. The basis for the communicative action relates to pretensions of validity rather than of power, which is external to language, and impedes the discussion of reasons and arguments. There was little disposition for dialogue and reciprocal recognition between the parties involved, with interdiction of a communicative situation in which there is symmetry of participation. The results revealed communicational weaknesses, thus requiring strategies that allow achieving communicative understanding among the professionals and promoting satisfactory patient follow-up between levels of care.

Health Evaluation; Health Care Levels;

Health Communication; Qualitative Research; Hermeneutics

\section{Resumen}

El objetivo del estudio fue comprender la influencia de las relaciones comunicacionales entre los profesionales de la red de atención a la salud en la coordinación entre niveles asistenciales. Estudio cualitativo con datos de investigación multicéntrica internacional Equity-LA II, utilizando el marco referencial teórico de la hermenéutica dialéctica. Se escucharon audios de 15 entrevistas a profesionales ( 7 médicos de la atención primaria y 5 de la atención especializada, y 3 profesionales de la atención primaria), en una red municipal en zona rural pernambucana, Brasil, en 2016. Las categorías mixtas se sometieron al análisis de contenido. Se reveló el no reconocimiento de la atención primaria como ordenadora del cuidado por casi todos los profesionales, y la percepción sobre la coordinación del cuidado desveló obstáculos relacionados con los desencuentros para establecer relaciones dialógicas. El saber sobre el papel del médico de la atención primaria es incompleto y su praxis vista con desconfianza por especialistas, lo que no sucede recíprocamente. Se percibió una relación interpersonal no dialógica, pautada en la relación asimétrica, reflejada en la postura autoritaria del especialista y de inferioridad de los médicos de la atención primaria. La base de la actuación comunicativa se refiere a las pretensiones de validez y no de poder, que es externo al lenguaje, $e$ imposibilita la discusión de razones y argumentos. Se evidenció la poca disponibilidad para el diálogo y reconocimiento recíproco entre los implicados, con prohibición de situaciones comunicativas donde existe simetría de participación. Los resultados revelaron fragilidades comunicacionales, siendo necesarias estrategias que permitan el alcance del entendimiento comunicativo entre los profesionales, promoviendo el seguimiento satisfactorio entre niveles asistenciales.

Evaluación en Salud; Niveles de Atención de Salud; Comunicación en Salud; Investigación Cualitativa; Hermenéutica
Recebido em 06/Fev/2020

Versão final reapresentada em 03/Jun/2020

Aprovado em 11/Jul/2020 